\title{
Intraoperative Dexmedetomidine Maintains Hemodynamic Stability and Hastens Postoperative Recovery in Patients Undergoing Transsphenoidal Pituitary Surgery
}

\section{Renu Bala ${ }^{1}$ Arvind Chaturvedi ${ }^{2}$ Mihir Prakash Pandia ${ }^{2}$ Parmod K. Bithal ${ }^{3}$}

\author{
${ }^{1}$ Department of Anaesthesiology and Critical Care, Pt BD Sharma \\ PGIMS, Rohtak, Haryana, India \\ 2Department of Neuroanaesthesia and Critical Care, AlIMS, \\ New Delhi, India \\ ${ }^{3}$ Department of Anesthesia, King Fahad Medical City, Riyadh, \\ Saudi Arabia
}

J Neurosci Rural Pract 2019;10:599-605
Address for correspondence Arvind Chaturvedi, MD, Department of Neuro Anaesthesia and Critical Care AlIMS, E-75, Ansari Nagar East, New Delhi 110 029, India (e-mail: c.arvind61@yahoo.co.in).

\begin{abstract}
Keywords

- anesthetic requirements

- dexmedetomidine

- hemodynamics

- pituitary surgery

- recovery

Background Transsphenoidal resection of pituitary tumors is the neurosurgical procedure of choice to excise most of the tumors of the sellar/suprasellar region. The main goals of anesthesia are maintenance of hemodynamic stability, provision of conditions that facilitate good surgical exposure, and a prompt and smooth emergence to allow neurological and visual assessment. Dexmedetomidine (Dex), a selective $\alpha-2$ agonist, is known to maintain cardiovascular stability and anxiolysis and provide pain relief. Therefore, we hypothesized that intraoperative Dex will attenuate hemodynamic response to nasal speculum (NS) insertion, decrease analgesic requirement, and hasten postoperative recovery.

Materials and Methods This prospective, randomized, double-blind, placebo-controlled study was conducted in 60 adult patients of either sex, American Society of Anesthesiologists status I or II undergoing elective pituitary surgery for excision of pituitary adenoma. Randomization was done into two groups; Group D ( $n=30)$ received Dex bolus $1 \mu \mathrm{g} / \mathrm{kg}$ over 10 minutes, followed by $0.5 \mu \mathrm{g} / \mathrm{kg} / \mathrm{h}$, and group control Group C $(n=30)$ received normal saline $(0.9 \%)$ in a similar manner. A standard anesthesia technique comprising fentanyl, propofol, rocuronium, sevoflurane, nitrous oxide, and oxygen was used. Intraoperative monitoring was uniform and standardized in all the patients; cardiovascular perturbations, if any, were noted and managed appropriately. After completion of surgery, tracheal extubation was performed, and emergence time, extubation time, modified Aldrete score, sedation, pain, time for first analgesic, nausea, vomiting, and shivering were recorded.

Results In both the groups, an increase in heart rate and blood pressure occurred at the time of laryngoscopy and intubation, NS insertion, and extubation, but it was more in Group C $(p<0.05)$. In Group D, intraoperative requirement of analgesic, neuromuscular relaxant, and inhalational anesthetic was lesser compared with Group $C$. Emergence time as well as visual analog scale at emergence was less in Group D.

Conclusions Intraoperative Dex infusion is a reasonable choice in patients undergoing transsphenoidal pituitary surgery.
\end{abstract}

License terms

()(1) $\Theta \circledast$ 


\section{Introduction}

Transsphenoidal surgical (TSS) resection of pituitary tumors accounts for as many as $20 \%$ of all intracranial operations performed for primary brain tumors at any academic center. ${ }^{1}$ Anesthetic management of these patients presents unique challenges to anesthesiologists. Strong nociceptor stimulation arising from nasal speculum (NS) insertion during this procedure produces severe hypertension and tachycardia, which in turn increase the surgical site bleeding obscuring surgical field. ${ }^{2.3}$ Moreover, such hemodynamic changes may be detrimental to a susceptible patient. Many techniques have been suggested to counter this noxious stimulation such as administering incremental doses of opioids, propofol, incremental concentrations of inhalational agents, and maxillary nerve block, but none has proven fully effective.,5 Moreover, some of these practices may delay recovery from anesthesia at the end of the surgical procedure. ${ }^{4}$

Early awakening from anesthesia after this neurosurgical procedure is of paramount importance, not only for neurological assessment of the patients but also to prevent any airway problems due to nasal packing as well as due to anatomical abnormalities of airway in acromegaly. Immediate postoperative visual acuity assessment of patients is a good guide to rule out hematoma compressing the optic chiasma and success of the surgical procedure. Thus, the main goals in technical solution procedure are prevention of disturbances of hemodynamics, provision of conditions conducive for good surgical exposure, and a prompt and smooth emergence from anesthesia to allow the early neurological and visual assessment. . $^{4}$

Dexmedetomidine (Dex) is a highly selective $\alpha-2$ agonist and has achieved widespread popularity in anesthesia as well as in intensive care practice. Owing to its central sympatholytic action, it attenuates intraoperative hypertensive episodes. It has established itself as a useful anesthetic adjuvant because it produces anxiolysis, reduces requirements of inhalational anesthetics as well as opioids, and is devoid of respiratory depression in the postoperative period. ${ }^{8,9}$

We hypothesized that intraoperative Dex administration in patients undergoing microscopic TSS pituitary surgery will maintain hemodynamic stability and decrease anesthetic requirements, thereby resulting in early recovery of these patients. The primary objective of our study was to evaluate the effects of Dex administration on hemodynamic disturbances during NS insertion, and secondary objectives were to assess its effects on intraoperative anesthetic and opioids' requirements and postoperative recovery.

\section{Materials and Methods}

After taking approval from ethics committee of the institution, 60 adult patients of both genders (age 18-65 years) and American Society of Anesthesiologists I/II physical status, scheduled to undergo nonemergent microscopic transsphenoidal pituitary tumor adenoma excision, were enrolled in this study. Exclusion criteria were patients with history of cerebrovascular disease, cardiovascular disease, pituitary apoplexy, chronic renal or liver disease, and patients on $\beta$-blockers. They were examined a day before the surgical procedure, and all the routine investigations were reviewed, and Allen's test was performed. The study was explained to the patients, and written informed consent was taken. They were explained of the visual analog scale (VAS) and were instructed for breathing through the mouth after the surgery. ${ }^{10}$ They were premedicated with $0.2 \mathrm{mg}$ glycopyrrolate, 30 minutes before transfer to operating room. They were randomly assigned to two groups according to computer-generated randomized chart and sealed opaque envelops. Routine monitoring comprising of electrocardiogram, noninvasive blood pressure (BP), and oxygen saturation probe and bispectral index (BIS) sensors were attached. Before induction of anesthesia, a bolus of $5 \mathrm{~mL} / \mathrm{kg}$ of normal saline was infused. Baseline values of heart rate (HR) and systolic, diastolic, and mean BP were noted (the mean of three values). In the Dex (D) group, a loading dose of $1 \mu \mathrm{g} / \mathrm{kg}$ was infused over 10 minutes. In the control (C) group, a similar syringe with the same volume of saline was infused over 10 minutes. Both the syringes were prepared by a consultant neuroanesthesiologist who was not involved in the study and neuroanesthesiologist administering them was blinded to the nature of agent in the syringes. Anesthesia was induced with fentanyl $2 \mu \mathrm{g} / \mathrm{kg}$ and a sleep dose of propofol (loss of response to verbal commands). After ensuring mask ventilation, rocuronium $1 \mu \mathrm{g} / \mathrm{kg}$ was administered to facilitate tracheal intubation. Posterior pharynx was packed with a roller gauze. Lungs were mechanically ventilated with oxygen nitrous mixture in a ratio of $1: 2$ and sevoflurane (minimum alveolar concentration [MAC] between 1.0 and 1.2) to maintain an end-tidal carbon dioxide tension around $36 \mathrm{~mm} \mathrm{Hg}$ and BIS between 40 and 60 by altering the sevoflurane concentration. In Group D, Dex was infused at the rate of $0.5 \mu \mathrm{g} / \mathrm{kg} / \mathrm{h}$, and in Group C, saline was infused at the same volume rate. Intraoperative analgesia was supplemented with fentanyl $1 \mu \mathrm{g} / \mathrm{kg}$ whenever HR and mean arterial pressure (MAP) increased by $20 \%$ above the baseline and persisted for at least 1 minute. A BIS value greater than 60 was treated with a bolus of $0.5 \mathrm{mg} / \mathrm{kg}$ of propofol, and the number of such boluses given was recorded. The surgeon inserted the NS after infiltration of nose and upper gingival surface with lignocaine containing 1:100,000 epinephrine. Speculum response resulting in an increase in BP if any, lasting more than 1 minute, was managed as below-mentioned protocol:

Fentanyl $1 \mu \mathrm{g} / \mathrm{kg}$ bolus was administered as the first line of treatment; if no improvement was observed, then, this was followed by a bolus of $0.5 \mathrm{mg} / \mathrm{kg}$ of propofol as the second line of management; and if this too also proved ineffective, then as a third line of treatment, labetalol was administered in boluses of $10 \mathrm{mg}$ each up to a maximum dose of $60 \mathrm{mg}$.

Similarly, hypotension (a decrease in MAP of more than 20\% lasting more than a minute) was managed by a fluid bolus as the first line of management; if this did not raise the BP level, then a vasopressor, mephentermine was administered in repeated boluses of $5 \mathrm{mg}$ each. Bradycardia (HR $<50$ beats/min persisting more than 1 minute) was treated by atropine boluses of $0.1 \mathrm{mg}$ each.

Rocuronium (10 mg) supplementation was given on initial appearance of waning of relaxant effect. Consumption of total dose of fentanyl, propofol, mephentermine, labetalol, atropine, and rocuronium and the number of fluid boluses were recorded. 
At the beginning of gingival suturing, infusion of Dex or saline was stopped, as well as sevoflurane was discontinued while nitrous was stopped only after completion of gingival suturing. Trachea was extubated after reversing the residual muscle paralysis with standard doses of neostigmine and glycopyrrolate.

Emergence time (time interval between discontinuation of nitrous oxide and time to open eyes spontaneously or to command) and time to extubate (time interval between stopping the nitrous and extubation) were noted. Hemodynamic response to extubation (that is peak rise in hemodynamic variables) was also recorded. Time taken from extubation to visual examination was also noted. Sedation level was assessed by Ramsey Sedation Score (RSS). ${ }^{11}$ VAS was also assessed soon after extubation. Quality of recovery was defined according to modified Aldrete score (MAS), and time to achieve full score was noted. ${ }^{12}$ Also recorded were complaints of nausea, vomiting, shivering, and agitation.

Patients were transferred to neurosurgical intensive care ward, and VAS was assessed every 15 minutes. Time for first requirement of analgesia was recorded (VAS > 3). Patients whose trachea could not be extubated were excluded from further analysis (postoperative).

\section{Statistical Analysis}

Sample size was computed to detect a difference of 20\% increase in MAP between the control group and the Dex group. The required sample size for $\alpha$ error of 0.05 and power of $80 \%$ was estimated to be 27 patients/group. We enrolled 30 patients/ group, assuming few dropouts from the study.

The statistical analysis was performed using statistical package for social sciences SPSS Windows 20 (SPSS Inc., Chicago, Illinois, United States). The demographic data were analyzed using the Student's $t$-test for continuous variables and chi-squared test for categorical variables. Intraoperative variables (duration of surgery, fluids transfused, and amount of drugs used) between the two groups were compared using the Student's $t$-test, while urine output and estimated blood loss were not normally distributed, hence compared using the Wilcoxon signed-rank test. Hemodynamic parameters and MAC value at a particular point of time between two groups were compared, using unpaired $t$ test and over a period of time using repeated-measures analysis followed by multiple comparisons with least square deviation. The incidence of side effects was analyzed with Fischer's exact test. Postoperative data were compared using unpaired $t$ test. Statistical significance between the groups was analyzed using post hoc comparison (multiple comparisons) by Bonferroni method. $p$-Value $<0.05$ was regarded as statistically significant.

\section{Results}

The demographic profile of the patients in two groups is shown in - Table $\mathbf{1}$ and is comparable. The history of hypertension was present in five patients in Group D and seven patients in Group C. The classification of tumor was done according to their size. The majority of patients in both the groups, 27 each (90\%), had macroadenoma. Following infusion of loading dose of Dex, BIS decreased to 85.6 (mean), but all the patients were arousable and maintained oxygen saturation. As per RSS, five patients had score of 1 while 15 and 10 patients had score of 2 and 3, respectively. The intraoperative details are shown in - Table 2. Blood loss was not significantly different between the groups. There was a significant decrease in intraoperative requirement of propofol, fentanyl, and rocuronium as depicted in - Table 2. Inhalational anesthetic (sevoflurane) requirement was also decreased significantly in Group D ( - Fig. 1). Hemodynamic alterations were encountered in both the groups, but it was more in Group C ( $\boldsymbol{-}$ Figs. $\mathbf{2}$ and $\mathbf{3}$ ). At the time of NS insertion, the increase in HR was $26 \%$ in Group $C$ as compared with $8.8 \%$ rise in Group D and an increase in MAP was $27 \%$ and 9.4\% in Groups C and D, respectively. Of 27 patients in Group $\mathrm{C}$ who presented with NS response, 8 patients received line 3 treatment (labetalol boluses). However, in Group D, none of the patient required line 3 treatment ( - Table 3 ). The patients in Group D had better and early recovery (-Table 4). Three patients in Group C were not extubated because of surgical reasons. They were electively ventilated in intensive care unit and successfully extubated on next day of surgery. Intraoperative bradycardia was seen in the patients in Group D and none in Group C, whereas hypotension occurred in two and five patients in Groups C and D, respectively. They were transient and responded to boluses of atropine and mephentermine. More number of patients in Group $C$ had postoperative nausea and vomiting (PONV) ( 6 vs. $2 ; p=0.25$ ) and shivering ( 4 vs. nil, $p=0.11$ ). Postoperative pain was complained by nine patients (33\%) in Group C and two patients (6.6\%) in Group D ( $p=0.01)$.

\section{Discussion}

There was significant reduction in induction dose of propofol, MAC of sevoflurane, consumption of rocuronium, and fentanyl in the Dex group. Furthermore, Dex infusion

Table 1 Patient characteristics

\begin{tabular}{|l|l|l|l|}
\hline Variable & Group C $(\boldsymbol{n}=\mathbf{3 0})$ & Group D $(\boldsymbol{n}=30)$ & $\boldsymbol{p}$-Value \\
\hline Age, y & $41 \pm 13.4$ & $37.2 \pm 11.0$ & 0.23 \\
\hline Height, $\mathrm{cm}$ & $164.5 \pm 8.9$ & $162.8 \pm 9.2$ & 0.47 \\
\hline Weight, $\mathrm{kg}$ & $71.2 \pm 14.8$ & $67.7 \pm 12.5$ & 0.33 \\
\hline Gender (male/female), \% & $17 / 13(56.7 / 43.3)$ & $16 / 14(53.3 / 48.7)$ & 0.79 \\
\hline ASA status (I/II), \% & $18 / 12(60 / 40)$ & $20 / 10(67.7 / 33.3)$ & 0.23 \\
\hline
\end{tabular}

Abbreviation: ASA, American Society of Anesthesiologists.

Note: Data are expressed as mean \pm SD; gender and ASA status is given as the number of patients and percentage. 
Table 2 Intraoperative details and drug requirement

\begin{tabular}{|l|l|l|l|}
\hline Variable & Group C $(\boldsymbol{n}=\mathbf{3 0})$ & Group D $(\boldsymbol{n}=30)$ & $\boldsymbol{p}$-Value \\
\hline Duration of surgery, min & $130.5 \pm 46.6$ & $114.6 \pm 43.6$ & 0.18 \\
\hline Nasal-speculum duration, min & $72.9 \pm 29.2$ & $67.5 \pm 23.4$ & 0.43 \\
\hline Fluid transfused, mL & $1970 \pm 446.5$ & $2066.6 \pm 537.1$ & 0.45 \\
\hline Urine output, mL & $445 \pm 329.1$ & $331.6 \pm 196.2$ & 0.11 \\
\hline Estimated blood loss, mL & $218 \pm 193.1$ & $153.3 \pm 147.9$ & 0.14 \\
\hline Propofol sleep dose, mg & $123.3 \pm 22.9$ & $80.6 \pm 21.4$ & $<0.0001$ \\
\hline Propofol supplementation, mg & $59.6 \pm 31.1$ & $30 \pm 14.1$ & 0.04 \\
\hline Fentanyl, $\mu$ g & $260.6 \pm 57.8$ & $182.5 \pm 39.7$ & $<0.0001$ \\
\hline Rocuronium, mg & $88.3 \pm 20.1$ & $73.6 \pm 16.0$ & 0.03 \\
\hline
\end{tabular}

Note: Data are expressed as mean \pm SD.

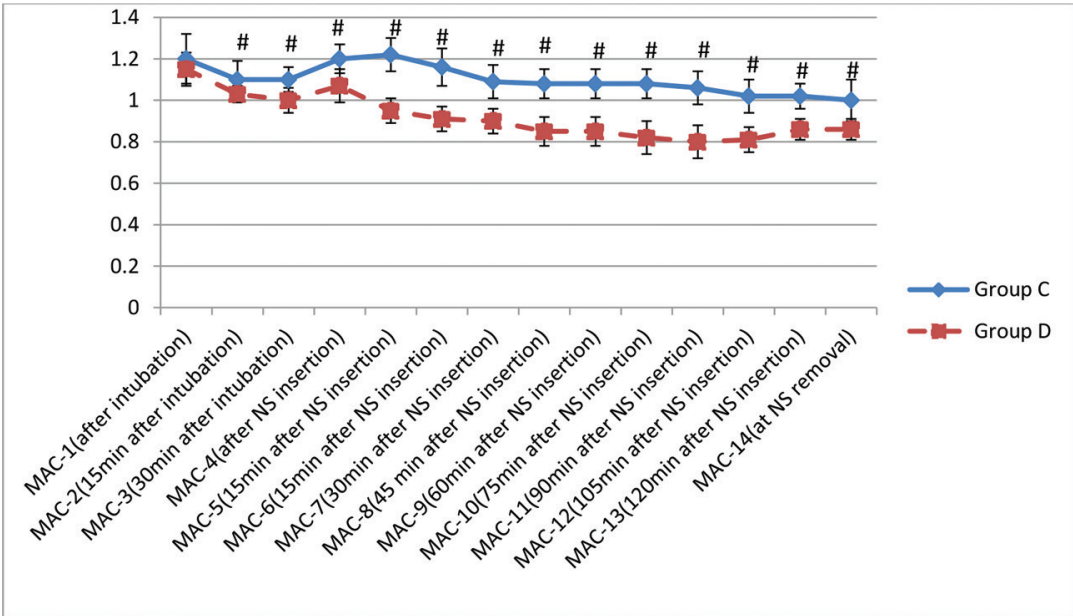

Fig. 1 Minimum alveolar concentration variation in two groups ( ${ }^{\sharp} p<0.05$, statistically significant). MAC, minimum alveolar concentration; NS, nasal speculum.

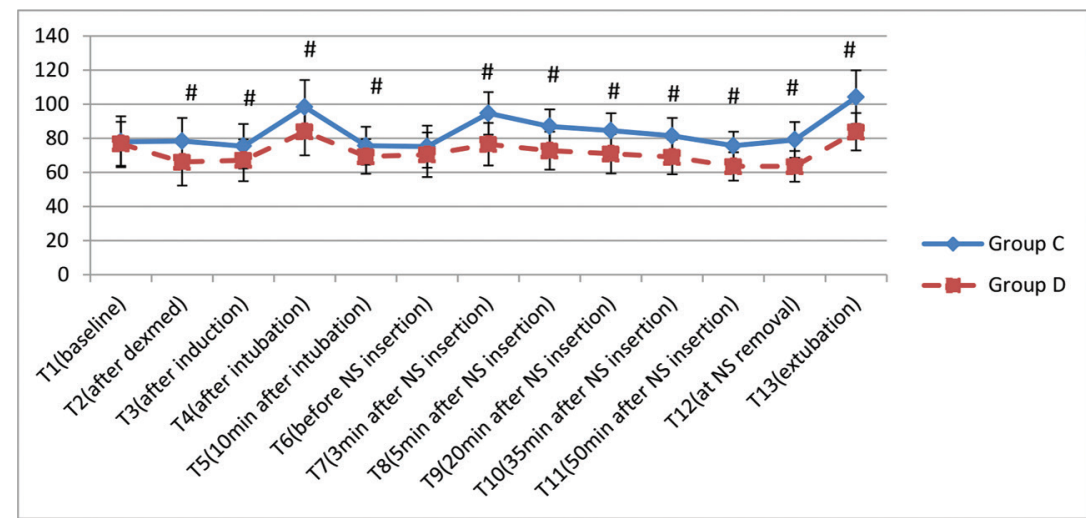

Fig. 2 Variation in heart rate (in beats/minute) at various time intervals. Data are expressed as mean \pm standard deviation $\left({ }^{\#} p<0.05\right.$, statistically significant). NS, nasal speculum.

produced stable hemodynamics during NS insertion compared with the control group. In the control group, significantly a greater number of patients required first and second line of antihypertensive measures to control high BP arising from NS insertion. More importantly, no patient in the Dex group needed third line of antihypertensive therapy, that is, labetalol, whereas $26.6 \%$ of the patients in the control group were administered third line of treatment.

Cardiovascular responses in the form of hypertension and tachycardia are frequently observed in various intracranial surgical procedures, and 50 to $90 \%$ of the patients require antihypertensive drugs perioperatively to restore BP level to 


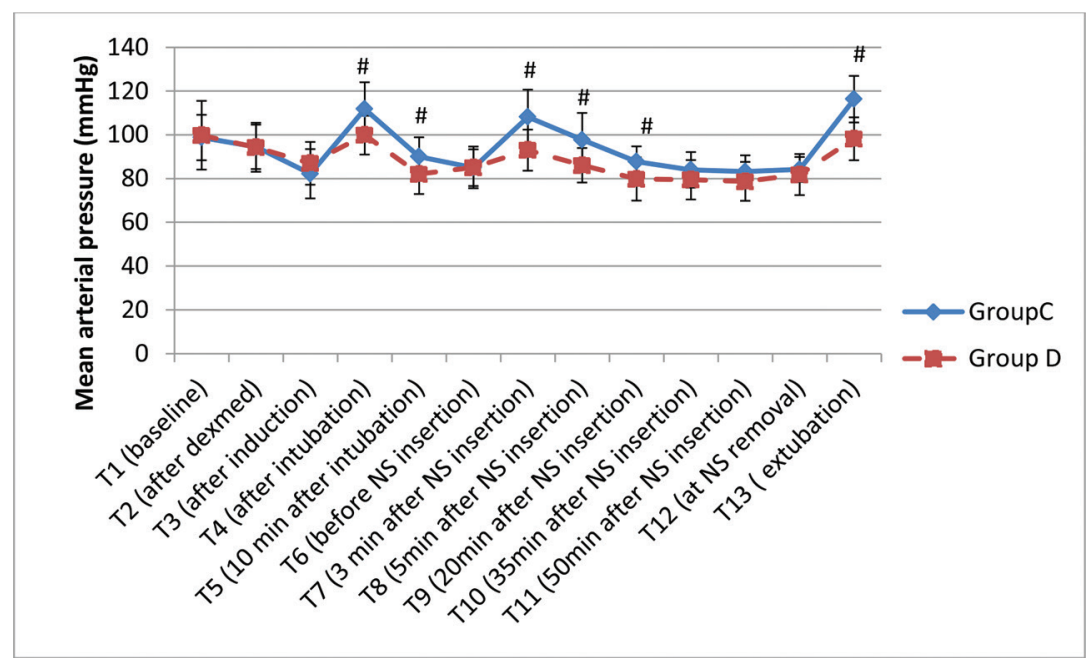

Fig. 3 Variation in mean arterial pressure (in $\mathrm{mm} \mathrm{Hg}$ ) at various time intervals. Data are expressed as mean \pm standard deviation $\left({ }^{\sharp} p<0.05\right.$, statistically significant). NS, nasal speculum.

Table 3 Nasal-speculum response

\begin{tabular}{|l|l|l|l|}
\hline Variable & Group C $(\boldsymbol{n}=30), \boldsymbol{n}(\%)$ & Group D $(\boldsymbol{n}=\mathbf{3 0}), \boldsymbol{n}(\%)$ & $\boldsymbol{p}$-Value \\
\hline Response present & $27(90)$ & $10(33.3)$ & $<0.001$ \\
\hline Line 1 treatment (fentanyl boluses) & $27(90)$ & $10(33.3)$ & $<0.001$ \\
\hline Line 2 treatment (propofol boluses) & $13(43.3)$ & $1(3.3)$ & $<0.001$ \\
\hline Line 3 treatment (labetalol boluses) & $8(26.6)$ & 0 & 0.02 \\
\hline
\end{tabular}

Note: Data are expressed as the number of patients and percentage within parentheses.

Table 4 Postoperative data

\begin{tabular}{|l|l|l|l|}
\hline & Group C $(\boldsymbol{n}=\mathbf{2 7})$ & Group D $(\boldsymbol{n}=\mathbf{3 0})$ & $\mathbf{p}$-Value \\
\hline Emergence time, min & $7.9 \pm 3.1$ & $5.0 \pm 2.4$ & $<0.0001$ \\
\hline Extubation time, min & $10.8 \pm 3.8$ & $7.7 \pm 3.1$ & 0.01 \\
\hline Ramsay sedation score & $2.7 \pm 0.5$ & $2.3 \pm 0.4$ & 0.05 \\
\hline MAS & $8.6 \pm 0.9$ & $9.1 \pm 0.7$ & 0.02 \\
\hline VAS & $3.8 \pm 1.2$ & $2.6 \pm 0.4$ & $<0.0001$ \\
\hline Time for visual examination, min & $2.8 \pm 0.9$ & $2.3 \pm 0.5$ & 0.17 \\
\hline Time for full MAS, min & $6.3 \pm 1.5$ & $5.8 \pm 0.9$ & 0.11 \\
\hline Time for 1st analgesic, min & $15 \pm 11.9$ & $80.6 \pm 36.6$ & $<0.0001$ \\
\hline
\end{tabular}

Abbreviations: MAS, modified Aldrete score; VAS, visual analog scale.

Note: Data are expressed as mean \pm SD.

normal. ${ }^{13,14}$ Microscopic TSS results in greater perturbations of $\mathrm{HR}$ and $\mathrm{BP}$ due to intense nociceptive stimuli arising during various stages of surgery. These responses can be blunted by increasing the depth of anesthesia and/or by administering opioids. However, both these maneuvers may cause hypotension, thereby compromising cerebral perfusion. Moreover, they may delay recovery following termination of anesthesia. ${ }^{4,6}$ In various general surgical and neurosurgical procedures, Dex has proved its efficacy in attenuating intraoperative increases in hemodynamics. Therefore, it has gained widespread acceptance in neurosurgical procedures. ${ }^{15-17}$ Both peripheral and central mechanisms are responsible for the hemodynamic effects of Dex. In the central nervous system, activation of $\alpha-2$ receptors leads to reduction in sympathetic outflow and an increase in vagal activity. In addition, Dex may also possess some peripheral ganglion-blocking action, which further enhances its sympatholytic effects. Our observations on intraoperative hemodynamic stability in presence of Dex are similar to various previous studies., ${ }^{418}$ Jan et al too came across similar results, while comparing oral clonidine with intravenous Dex infusion in patients undergoing transsphenoidal resection of pituitary tumors. Although hemodynamic fluctuations occurred in both groups, patients in the Dex group had better hemodynamic stability. ${ }^{19}$ 
The exact mechanisms of propofol-sparing effects of Dex are unclear; it is speculated that peripheral vasoconstrictor effects of Dex retard redistribution of propofol, thereby augmenting its actions at the effect site, despite administering a lower induction dose. Besides, owing to its sedative effect, Dex reduces the induction dose of Propofol, which in turn leads to a lower incidence of hypotension.

By virtue of its $\alpha-2$ agonist actions that inhibit noradrenergic transmission, Dex reduces the requirement of inhalational anesthetic agents. ${ }^{9}$ In our study too, Dex reduced the MAC of sevoflurane by $14 \%$, which is in consonance with the previous studies. ${ }^{20,21}$ We monitored anesthetic depth with BIS, an accepted tool for monitoring depth of anesthesia. ${ }^{22}$ Similar to the previous study of Memis et al, we too noted a reduced requirement of rocuronium in patients of the Dex group, probably resulting from an altered pharmacokinetics of rocuronium presence of Dex. ${ }^{23}$ Reduced requirements of sevoflurane and rocuronium by the Dex infusion will be of tremendous benefit to a neurosurgical patient because a faster recovery from anesthesia would aid the neurosurgeon to conduct an early neurological examination of the patient.

Fentanyl requirement was reduced by $30 \%$ in the Dex group. Animal studies have shown synergistic analgesic action of Dex and fentanyl. ${ }^{24}$ However, this observation in our study was at variance with a similar study published by Gopalakrishna et al. ${ }^{4}$ The latter study differed from our study in that the authors avoided nitrous oxide unlike our study. It has been previously reported that administration of Dex in the presence of nitrous is likely to provide enhanced and durable analgesia. ${ }^{25}$ This may explain different observations on requirement of fentanyl in the two studies. Salimi et al found that intraoperative infusion of $0.6 \mu \mathrm{g} / \mathrm{kg} / \mathrm{h}$ in pituitary surgeries reduced intraoperative propofol and fentanyl requirement similar to our results, but they also observed reduced intraoperative blood loss in the Dex group contrary to our findings. Surgeons' satisfaction that was not evaluated in our study was also better in the Dex group. ${ }^{18}$

The primary requirements of neurosurgical anesthesia are early emergence and tracheal extubation. Both these parameters were significantly shorter in the Dex group compared with the control group. Decreased consumption of sevoflurane, rocuronium, and fentanyl in the Dex group was the reason for faster emergence and extubation. On the other hand, Gunes et al reported delayed recovery following termination of anesthesia in patients administered Dex during intracranial surgery. ${ }^{26}$ However, these authors infused a higher dose of Dex $(0.6-1.2 \mu \mathrm{g} / \mathrm{kg} / \mathrm{h})$ in intracranial surgery compared with $0.5 \mu \mathrm{g} / \mathrm{kg} / \mathrm{h}$ of Dex in our patients of TSS procedures. Therefore, the two studies cannot be compared with each other. Following tracheal extubation, RSS was comparable at all the time points in the postoperative period, and the time to vision examination was comparable. Since vision examination requires patient's full cooperation, similar RSS after extubation resulted in similar time interval for vision examination. Most of the patients in the Dex group woke up from anesthesia pain free and had delayed request for first analgesic in the postoperative period. This emphasizes the analgesic effect of Dex, which may decrease opioids' requirements postoperatively.

Although MAS immediately after extubation was statistically higher in the Dex group but the difference was significant clinically, similar MASs were reported by Unlugenc et al also in their study, the protocol of which, however, was different from our study, and it was conducted in nonneurosurgical patients. ${ }^{27}$ PONV was more in the control group. Alpha-2 agonists reduce salivary flow and gastrointestinal motility that can contribute to decreased nausea and vomiting in patients receiving Dex. Furthermore, there was decreased opioid and inhalational anesthetic consumption in the Dex group that could have also attributed to reduced PONV. The exact mechanism remains, however, unclear. ${ }^{28,29}$ Dex has been used to maintain stable hemodynamics in endoscopic transnasal pituitary surgeries although our patient group was for microscopic surgeries. The authors noted greater incidences of hypotension and bradycardia in the Dex group. ${ }^{30}$

There are quite a few limitations in our study. First, when we conducted this study, the microscopic TSS for pituitary adenoma excision was in vogue, a technique that has gradually been replaced by endoscopic technique. The latter technique does not require NS insertion, yet requires turbinectomy (which is not as stressful as NS insertion). Although significant hemodynamic perturbations may result during endoscopic procedure too, we cannot comment on the benefits of Dex in this setting. However, other benefits of Dex infusion will accrue in endoscopically performed TSS also. Second, we cannot comment on impact of Dex infusion, if any, in patients with cardiovascular diseases since we excluded this group of patients from our study. Third, we topped up rocuronium based on capnography, which is not a proper technique for monitoring muscle relaxation. Moreover, total sevoflurane consumption could have been measured. Fourth, stress hormone levels' (such as cortisol) measurement, a better marker of stress, should have been measured during NS insertion, but we were handicapped by the absence of this laboratory facility. Finally, we compared Dex with placebo, which had obvious results. Comparison of dexmedetomidine with other drugs can be performed in future trials.

\section{Conclusion}

Our study suggests that intraoperative infusion of Dex in patients undergoing microscopic TSS offers a valuable strategy to blunt the hemodynamic perturbations during NS insertion and promotes quick recovery by reducing intraoperative requirement of sevoflurane, fentanyl, and rocuronium.

\section{Funding \\ None.}

\section{Conflict of Interest}

None declared. 


\section{References}

1 Horvat A, Kolak J, Gopcević A, Ilej M, Zivko G. Anesthetic management of patients undergoing pituitary surgery. Acta Clin Croat 2011;50(2):209-216

2 Nemergut EC, Dumont AS, Barry UT, Laws ER. Perioperative management of patients undergoing transsphenoidal pituitary surgery. Anesth Analg 2005;101(4):1170-1181

3 Lim M, Williams D, Maartens N. Anaesthesia for pituitary surgery. J Clin Neurosci 2006;13(4):413-418

4 Gopalakrishna KN, Dash PK, Chatterjee N, Easwer HV, Ganesamoorthi A. Dexmedetomidine as an anesthetic adjuvant in patients undergoing transsphenoidal resection of pituitary tumor. J Neurosurg Anesthesiol 2015;27(3):209-215

5 Chadha R, Padmanabhan V, Rout A, Waikar HD, Mohandas K. Prevention of hypertension during trans-sphenoidal surgerythe effect of bilateral maxillary nerve block with local anaesthetics. Acta Anaesthesiol Scand 1997;41(1 Pt 1):35-40

6 Ali Z, Prabhakar H, Bithal PK, Dash HH. Bispectral index-guided administration of anesthesia for transsphenoidal resection of pituitary tumors: a comparison of 3 anesthetic techniques. J Neurosurg Anesthesiol 2009;21(1):10-15

7 Gupta D, Srivastava S, Dubey RK, Prakash PS, Singh PK, Singh U. Comparative evaluation of atenolol and clonidine premedication on cardiovascular response to nasal speculum insertion during trans-sphenoid surgery for resection of pituitary adenoma: a prospective, randomised, double-blind, controlled study. Indian J Anaesth 2011;55(2):135-140

8 Yazbek-Karam VG, Aouad MM. Perioperative uses of dexmedetomidine. Middle East J Anaesthesiol 2006;18(6):1043-1058

9 Herbert BA, Ramaciotti PM, Ferrari F, et al. [The use of dexmedetomidine in neurosurgery.]. Rev Bras Anestesiol 2007;57 (2):223-231

10 Katz J, Melzack R. Measurement of pain. Surg Clin North Am 1999;79(2):231-252

11 Ramsay MA, Savege TM, Simpson BR, Goodwin R. Controlled sedation with alphaxalone-alphadolone. BMJ 1974;2 (5920):656-659

12 Aldrete JA. The post-anesthesia recovery score revisited. J Clin Anesth 1995;7(1):89-91

13 Struitis M, Kroin J, Swamidoss C, Moric M. Effects of intraoperative dexmedetomidine infusion on hemodynamic stability during brain tumor resection. Anesthesiology 2002;98:A-310

14 Tanskanen PE, Kyttä JV, Randell TT, Aantaa RE. Dexmedetomidine as an anaesthetic adjuvant in patients undergoing intracranial tumour surgery: a double-blind, randomized and placebo-controlled study. Br J Anaesth 2006;97(5):658-665

15 Yildiz M, Tavlan A, Tuncer S, Reisli R, Yosunkaya A, Otelcioglu S. Effect of dexmedetomidine on haemodynamic responses to laryngoscopy and intubation: perioperative haemodynamics and anaesthetic requirements. Drugs R D 2006;7(1):43-52

16 Uyar AS, Yagmurdur H, Fidan Y, Topkaya C, Basar H. Dexmedetomidine attenuates the hemodynamic and neuroendocrinal responses to skull-pin head-holder application during craniotomy. J Neurosurg Anesthesiol 2008;20(3):174-179
17 Bekker A, Sturaitis M, Bloom M, et al. The effect of dexmedetomidine on perioperative hemodynamics in patients undergoing craniotomy. Anesth Analg 2008;107(4):1340-1347

18 Salimi A, Sharifi G, Bahrani H, et al. Dexmedetomidine could enhance surgical satisfaction in trans-sphenoidal resection of pituitary adenoma. J Neurosurg Sci 2017;61(1):46-52

19 Jan S, Ali Z, Nisar Y, et al. A comparison of dexmedetomidine and clonidine in attenuating the hemodynamic responses at various surgical stages in patients undergoing elective transnasal transsphenoidal resection of pituitary tumors. Anesth Essays Res 2017;11(4):1079-1083

20 Nunes RR, Cavalcante SL. Influence of dexmedetomidine upon sevoflurane end-expiratory concentration. Evaluation by bispectral index, suppression rate and electroencephalographic power spectral analysis. Rev Bras Anestesiol 2002;52(2):133-145

21 Magalhães E, Govêia CS, Ladeira LC, Espíndola BV. [Relationship between dexmedetomidine continuous infusion and end-tidal sevoflurane concentration, monitored by bispectral analysis.]. Rev Bras Anestesiol 2004;54(3):303-310

22 Ellerkmann RK, Liermann VM, Alves TM, et al. Spectral entropy and bispectral index as measures of the electroencephalographic effects of sevoflurane. Anesthesiology 2004;101(6):1275-1282

23 Memis D, Turan A, Karamanilioglu B, Seker S, Pamakcu Z. Dexmedetomidine reduces rocuronium dose requirement in sevoflurane anaesthesia. Curr Anaesth Crit Care 2008;19:169-174

24 Horváth G, Szikszay M, Rubicsek G, Benedek G. An isobolographic analysis of the hypnotic effects of combinations of dexmedetomidine with fentanyl or diazepam in rats. Life Sci 1992;50(23):PL215-PL220

25 Dawson C, Ma D, Chow A, Maze M. Dexmedetomidine enhances analgesic action of nitrous oxide: mechanisms of action. Anesthesiology 2004;100(4):894-904

26 Gunes Y, Gunduz M, Ozcengiz D, Ozbek H, Isik G. Dexmedetomidine-remifentanil or propofol-remifentanil anaesthesia in patients undergoing intracranial surgery. Neurosurg Q 2005;15:122-126

27 Unlugenc H, Gunduz M, Guler T, Yagmur O, Isik G. The effect of pre-anaesthetic administration of intravenous dexmedetomidine on postoperative pain in patients receiving patient-controlled morphine. Eur J Anaesthesiol 2005;22(5):386-391

28 Massad IM, Mohsen WA, Basha AS. Al-Zaben KR, Al-Mustafa MM, Alghanem SM. A balanced anesthesia with dexmedetomidine decreases postoperative nausea and vomiting after laparoscopic surgery. Saudi Med J 2009;30(12):1537-1541

29 Afonso J, Reis F. Dexmedetomidine: current role in anesthesia and intensive care. Rev Bras Anestesiol 2012;62(1):118-133

30 Soliman R, Fouad E. The effects of dexmedetomidine and magnesium sulphate in adult patients undergoing endoscopic transnasal transsphenoidal resection of pituitary adenoma: a double-blind randomised study. Indian J Anaesth 2017;61(5):410-417 